AperTO - Archivio Istituzionale Open Access dell'Università di Torino

\title{
JEM-EUSO: Meteor and nuclearite observations
}

\section{This is a pre print version of the following article:}

Original Citation:

\section{Availability:}

This version is available http://hdl.handle.net/2318/1636726

since 2017-05-19T18:59:28Z

Published version:

DOI:10.1007/s10686-014-9375-4

Terms of use:

Open Access

Anyone can freely access the full text of works made available as "Open Access". Works made available under a Creative Commons license can be used according to the terms and conditions of said license. Use of all other works requires consent of the right holder (author or publisher) if not exempted from copyright protection by the applicable law. 


\title{
JEM-EUSO: Meteor and nuclearite observations
}

\author{
The JEM-EUSO Collaboration
}

Received: date / Accepted: date

\begin{abstract}
Meteor and fireball observations are important to derive the inventory and physical characterization of the population of small solar system bodies orbiting in the vicinity of the Earth. After decades of ground-based activities, the proposed JEM-EUSO mission has some chances to become the first operational space-based platform having among its scientific objectives the observation of fireball and meteor events. The observing strategy developed to detect these phenomena, which are eminently "slow" events with respect to the extremely energetic cosmic ray events which are the primary objective of the mission, can prove to be very suitable also for the possible detection of nuclearites, an exciting possibility which enhances the overall scientific rationale of JEM-EUSO, and suggests that the planned observation of slow events may be very interesting in many respects.
\end{abstract}

Keywords Meteors · Nuclearites · JEM-EUSO · Space Detectors

M. Bertaina (corr. author)

Dipartimento di Fisica, Università di Torino \& INFN Torino, via P. Giuria 1, I-10125 Torino, Italy

Tel.: +39-011-6707492

Fax: +39-011-6707493

E-mail: bertaina@to.infn.it

A. Cellino (corr. author)

INAF-Osservatorio Astrofisico di Torino, Strada Osservatorio 20, I-10025 Pino Torinese (TO), Italy

Tel.: +39-011-8101933

Fax: +39-011-8101930

E-mail: cellino@oato.inaf.it

F. Ronga (corr. author)

Istituto Nazionale di Fisica Nucleare - Laboratori Nazionali di Frascati, Via E. Fermi 40, I-00044 Frascati, Italy

Tel.: +39-06-94032914

Fax: +39-06-94032900

E-mail: francesco.ronga@lnf.infn.it 


\section{Introduction}

JEM-EUSO, namely the proposed Extreme Universe Space Observatory on board the Japanese Experiment Module of the International Space Station (ISS) $[16,27,9]$ is currently under study, and is designed to be a great example of a multi-disciplinary mission, able to produce results of utmost importance for a wide and heterogeneous scientific community which includes theoretical and experimental physicists, high-energy astrophysicists, solar system specialists and experts of atmospheric phenomena. This unusually wide range of disciplines which contribute to the science case of JEM-EUSO is certainly one of the major strengths of this mission concept.

For what concerns the detection of meteor phenomena, one should be aware that large networks of ground-based observing stations have been established since a long time in many countries, including Europe, USA, Japan and Australia, to detect and record these events. Several ground-based networks for meteor observations are still active and include both amateur and professional observers, using a variety of detectors, ranging from old-fashioned photographic cameras to state-of-the-art electronic detectors. Moreover, meteors are also detected at radio wavelengths, as demonstrated as an example by the Bologna-Modra forward-scatter system [29].

Although all the above-mentioned ground-based facilities have great merits and have been successfully operational since many years, this does not mean that they are not affected by a number of problems. First, the covered sky area is in any case forcedly limited. Even in the best cases, a single groundbased network for meteor observations covers less than $1 \%$ of the Earth's surface. Second, the efficiency of meteor optical observations from the ground is seriously affected by weather conditions, including primarily the presence of clouds. As a consequence, the ideal goal of ground-based networks, namely the simultaneous detection of some fireball ${ }^{1}$ by different observers, in such a way as to be able to accurately determine the path of the body and eventually recover some meteorite reaching the ground, has been rarely achieved for many years.

On the other hand, meteor events are in principle best detectable from space-based facilities [13]. This includes both the light emitted at visible wavelengths, and the thermal infrared radiation produced by the heating of the meteoroid material all along its path through the atmosphere. Infrared observations are certainly possible also from the ground, but space-based detectors work much more efficiently in the infrared. This is trivially true when sources above the atmosphere are concerned, but also in cases of meteor phenomena, in which the phenomena occur in the high layers of the atmosphere, spacebased infrared detectors suffer in any case from much reduced atmospheric extinction with respect to ground-based facilities.

\footnotetext{
1 The term fireball is usually adopted to indicate extremely bright meteors, as it will be explained below.
} 
Space-based sensors have a number of other obvious advantages. They can cover wider areas of sky, and, being located above the atmosphere, they are not limited by weather conditions, and can operate in principle also in day time, at least for the detection of bright events. In spite of these advantages, meteor and fireball detections by space-based facilities have not been routinely reported so far. The reason is that existing satellites are usually equipped to monitor phenomena having much longer durations than the very few seconds which generally characterize meteor phenomena. As a consequence, these devices do not detect and record meteor events. On the other hand, satellites which are equipped with sensors suited for fireball detections have usually military purposes. These purposes do not include the scientific study of meteor events, and the data produced by their detectors are usually classified. As a consequence, meteor detections by military satellites are generally not made public and are eventually lost.

After decades of ground-based activities, the times seem now mature to plan the development of a new generation of dedicated, space-based facilities for the detection of meteors. JEM-EUSO is a very good candidate to become the first example of a space mission producing important data for meteor studies, as will be seen in the next Sections.

It should also be noted that a very interesting by-product of the observations of meteor-like objects might potentially be the detection of super-heavy exotic particles that could be important components of the so-called dark matter in our Universe. In particular, some neutral strange-quark material particles, called nuclearites, can produce UV emissions which could be comparable to meteor signals. We estimate that the large aperture of the JEM-EUSO telescope can produce an improvement of about three orders of magnitude over the existing limits for the detection of high-mass nuclearites in only 24 hours of operations.

The present paper is organized as follows: in section 2 we summarize some relevant notions concerning meteors. In Section 3 we do the same for nuclearites. In Section 4 we describe in general terms the focal plane assembly of JEM-EUSO, in order to explain the general principles of data acquisition. In Section 5 we describe the body of simulations which have been carried out so far in order to design a reliable strategy for the observations of slow events, primarily meteors. A summary of the obtained results is given in Section 6. In that Section, we focus our attention also on the subject of nuclearites, and we explain why we believe that the general observing strategy designed for the observation of meteors is in principle suitable also for the possible detection of this exotic state of matter. The general conclusions of our current work and the perspectives for future refinements are discussed in Section 7. 


\section{Brief outline of meteor phenomena}

The presence of a large population of micro-meteoroids in space is indicated by the existence of the so-called zodiacal light, consisting of sunlight reflected by small dust particles orbiting the Sun near the ecliptic plane.

The vast majority of the extraterrestrial bodies hitting the Earth are in fact sub-mm dust particles, which can become visible as meteors in the night sky.

The typical beginning heights of meteor phenomena is between 75 and 120 $\mathrm{km}$. The end points are generally at heights between 30 and $100 \mathrm{~km}$. The duration of the events range typically between 0.5 and a few seconds. These are therefore slow events for JEM-EUSO, as we will see below. The brightness varies over a huge interval, including events which are not visible by the human eye up to extremely bright (and much more unusual) events, which may be well visible even during day time. The latter correspond to much larger meteoroid sizes and masses.

About $75 \%$ of meteor events are sporadic, whereas one fourth of the events are genetically associated in a number of meteoroid streams which produce meteor showers in well defined epochs of the year. This is due to the fact that each meteoroid stream is formed by bodies having very similar orbits, likely produced by low velocity ejection from a common parent body, usually some comet. Many known meteor showers (like the Lyrids and the Perseids to mention only a few of them) are known to be associated with a parent comet. In the case of the Geminids, the parent body is an object previously classified as an asteroid, (3200) Phaeton.

The population of small Solar system bodies with orbits sweeping the region of the inner planets, however, does not include only micro-meteoroids. Much larger bodies, though less abundant, do exist. It is known that, apart from comets, a population of so-called near-Earth asteroids (NEA) have orbits partly or totally interior to the orbit of Mars. The size distribution of the NEAs is described by a power-law (smaller objects being increasingly more abundant). It is currently estimated that about 1000 NEAs with sizes of the order of $1 \mathrm{~km}$ exist. The number of bodies with sizes of some tens of meters should be of the order of 1 Million. Figure 1 shows the estimated NEA size distribution as it was presented a few years ago in the NASA Report to the US Congress about Near-Earth Object Survey and Deflection Analysis of Alternatives. Note that the Figure shows, in addition to the size and expected number of objects (both in log scale) also estimates of the absolute magnitudes and impact frequencies of objects of different sizes, as well as an estimate of the delivered impact energies in case of a collision with our planet. Note also that the absolute magnitude of an asteroid is defined as the visual (that is, measured in the standard $V$ filter) magnitude that would be measured if the object was seen at unit distance from both the Sun and the observer, and in condition of ideal solar opposition. This definition takes into account that the apparent brightness of these objects varies (trivially) as a function of distance, and (less trivially) as a function of illumination conditions. Note also that, in 


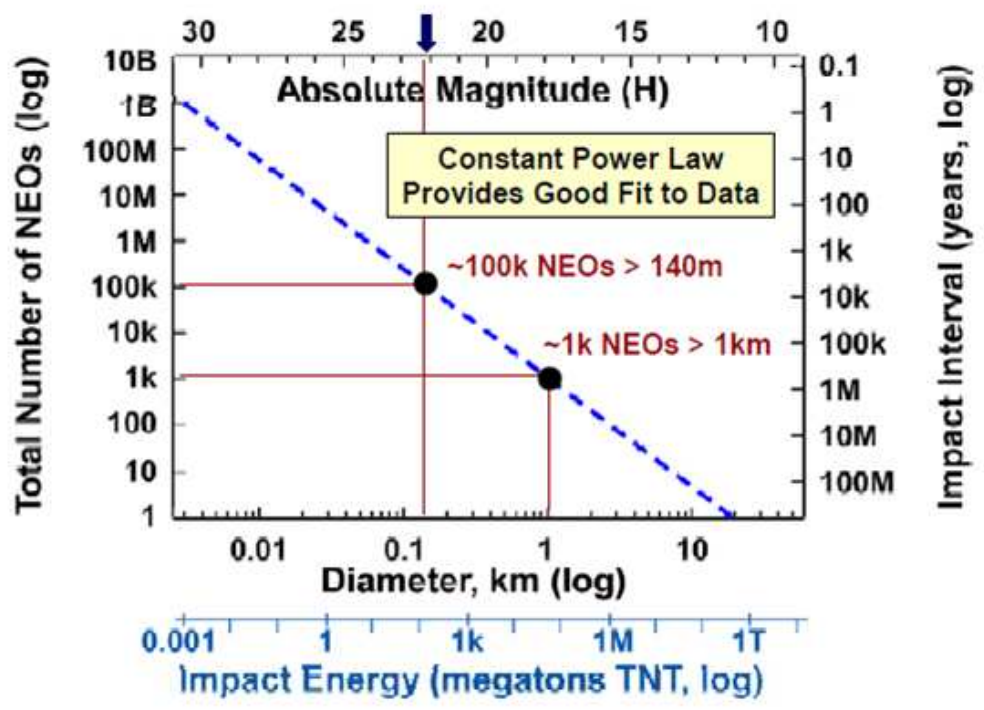

Fig. 1 The size and absolute magnitude distributions of near-Earth objects according to the 2007 NASA Report to the US Congress (for a definition of the absolute magnitude of asteroids, see the text)

the case of meteors, another definition of absolute magnitude holds: in particular, the absolute magnitude of a meteor is the apparent magnitude which would be measured by a ground-based observer if the meteor was seen at the zenith and at a distance of $100 \mathrm{~km}$ above the observer. It is clear, in fact, that the apparent brightness of a meteor depends on the distance, and also much on the atmospheric absorption, which is different at different angular distances from the zenith.

Objects with sizes of the order of some tens of meters or larger are called asteroids, while the word meteoroid applies at smaller sizes. These are very faint objects, that can hardly be detected by means of telescopes, so they reveal their existence mostly when they collide with the Earth's atmosphere and become bright. As a matter of fact, whenever the impacting body's size is larger than some limit depending on the entry velocity vector (about $20 \mathrm{~cm}$ for a velocity entry of $15 \mathrm{~km} / \mathrm{sec}$ from the zenith direction), the resulting meteor can be extremely bright. When the apparent brightness of a meteor reaches a magnitude around -4 or brighter at visible wavelengths, it is called fireball, or bolide, corresponding to impactor masses which may vary approximately from 0.1 up to 1000 kilograms. When the magnitude reaches or exceeds -17 , corresponding to impacting bodies above $1,000 \mathrm{~kg}$ in mass, the term superbolide is also used [13].

Meteoroids hit the Earth's atmosphere at hypersonic velocities ranging mostly between about 12 and $72 \mathrm{~km} / \mathrm{sec}$. Depending on the entry velocity 
and the mass of the meteoroid, or micro-meteoroid, different phenomena are then produced. All of them, in practice, are a consequence of the conversion of the kinetic energy of the impacting body into other forms of energy. As a general rule, a release of ions and free electrons occurs along the meteoroid path through the atmosphere. This is due to collisions of the material of the meteoroid's surface with atoms and molecules of the atmosphere. Visible light is then produced by de-excitation of these ions. In less frequent cases, corresponding to the most energetic events, additional detection of acoustic and infrasonic blast wave effects is also possible.

The most common outcome of the entry of a meteoroid in the Earth's atmosphere is the meteor phenomenon, which is produced by tiny bodies having sizes which, depending on the entry velocity, are typically above some fraction of a $\mathrm{mm}$ [12]. After a preliminary heating at heights between 300 and 100 $\mathrm{km}$ (preheating phase), a phase called ablation follows, in which the surface material starts to sublimate and a layer of hot vapor is produced around the body, which is heated up to temperatures well above $2000 \mathrm{~K}$. Excited states of the ions in this surrounding layer are produced and emit light at characteristic lines while they lose energy and are de-excited. The beginning height of this phenomenon ranges generally between 75 and $120 \mathrm{~km}$. This process continues until the body is completely disintegrated, generally at heights between 30 and $100 \mathrm{~km}$, after traveling over a distance which may range from several kilometers up to few tens of kilometers. A typical meteor ends up losing all its mass, though not radically changing its velocity: the latter generally decreases by an amount between several percent up to a few tens of percent, depending on the range of heights covered by its path through the atmosphere (the attrition becoming larger at lower heights), and depending also in some complicated way on the shape (cross section) of the body itself. The duration of the phenomenon is generally between 0.5 and 3 seconds. Because ions and free electrons are produced in a ionized column along the path of the body in the atmosphere, it is possible to detect these events also by means of radar techniques.

In particular conditions, when the impactor is sufficiently large and resistant, the meteoroid can survive the phase of ablation, and it is slowed down until it reaches a state of free fall. A phase of so-called dark flight can be reached, and what remains of the meteoroid eventually reaches the ground as a meteorite. In extreme cases, finally, the meteoroid can be so big that its velocity is only weakly reduced by interaction with the atmosphere, no dark-fly, free fall phase occurs, the body hits violently the ground at hypersonic speed producing an impact crater, or possibly a tsunami in case of fall on the water. These extremely rare events, which can have been responsible of episodes of mass extinction of the Earth's biosphere in the past, are clearly beyond the scopes of this article.

According to [13], over average timescales of the order of 100 years, most of the mass delivered to the Earth by colliding interplanetary bodies comes from super-bolides, having individual masses between $10^{3}$ and $10^{7} \mathrm{~kg}$, and typical sizes of the order of 10 meters. Bodies in this size range are the least 
known population of minor bodies in our Solar system. The number of events involving bodies in this size range is thought to be larger than 50 events per year, justifying a systematic observational effort.

Apart from the very rare and hugely destructive impacts producing craters on the Earth's surface and regional or global devastations, bolides and superbolides represent the most spectacular events of collision with extraterrestrial material. Since several decades a big effort has been made in order to be able to detect and record the maximum possible number of these events, with the general purpose of being able to determine the three-dimensional entry velocity vector of the bodies, in order to derive their pristine heliocentric orbits, to obtain some information about their composition, derivable from spectra, to derive hints on the ablation mechanisms based on the observed meteor lightcurve ${ }^{2}$, and to record the path in the atmosphere in order to compute also the likely regions of fall of possible associated meteorites. The derivation of the inventory and size distribution of the bodies which can intersect the orbit of the Earth with a non-zero probability of collision with our planet, has been since a long time a high priority task of modern Planetary Science. Apart from obvious considerations about mitigation of the impact hazard for the terrestrial biosphere, this is also a challenging theoretical problem, with important implications for our understanding of the orbital and physical evolution of the minor bodies of our Solar System.

At much smaller sizes, typical of micro-meteoroids, the inventory of the population and the typical flux of Earth impactors is even more difficult to determine. Some estimates have been done based either on the observed numbers of micro-craters on the surface of the Moon, or on spacecraft-based measurements [18]. Figure 2, adapted from a a plot published in the quoted [18] paper, shows a plot of the expected number per second of micro-meteoroid impacts occurring in the JEM-EUSO FoV as a function of micro-meteoroid mass. At very small masses some discrepancies exist between existing models, but it is clear that the number of events is in any case expected to be very high. According to Figure 2, the number of expected events by micro-meteoroids with masses of $0.001 \mathrm{~g}$ in the JEM-EUSO FoV is of the order of 1 per second. The apparent brightness of these events is faint, however, so to detect them it will be necessary to exploit limited interval of times when JEM-EUSO looks over very dark regions.

We want also to note that, apart from purely scientific issues, a spacebased system for fireball surveillance could have also some more immediately practical purposes. It is known, for instance, that the clouds of dust released by fireball events can be a hazard for aircraft. For these and other reasons, a prompt detection of these events could be of the highest importance also for mitigation of possible dangers for human beings in particular circumstances.

\footnotetext{
2 The meteor lightcurve is the recorded variation of the apparent meteor brightness as a function of time
} 


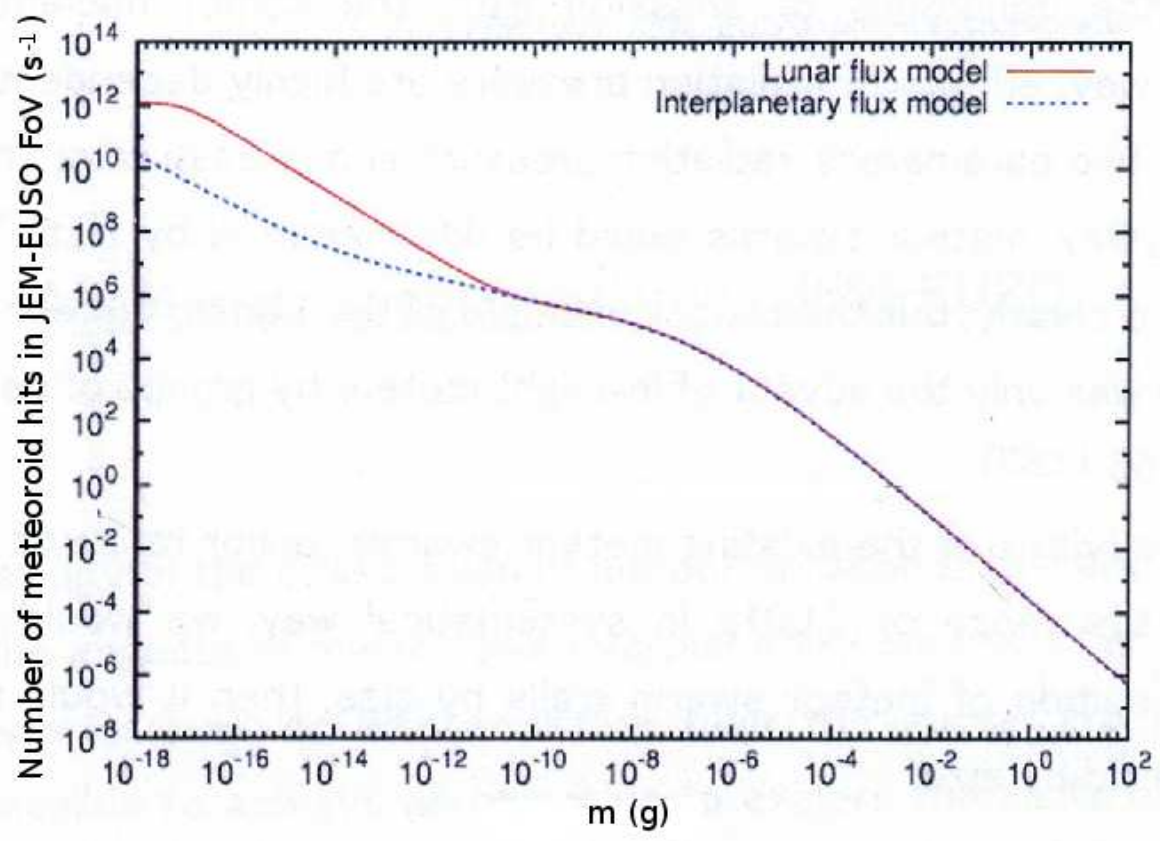

Fig. 2 Number of expected impacts per sec of micro-meteoroids in the JEM-EUSO FoV as a function of impactor mass, in grams. Figure adapted from [18]

\section{Brief outline of nuclearites and other super-heavy particles}

During the last decade a very large experimental and theoretical effort has been devoted to understand the problem of dark matter (DM). There is a compelling evidence of the existence of DM at all astrophysical length scales. Astrophysics and the "standard cosmology" require $84 \%$ of matter to exist in the form of DM. The local DM density in our galactic halo should be of the order of $0.3 \mathrm{GeV} / \mathrm{cm}^{3}$, and the DM speed should have values typical of galactic halos, around $270 \mathrm{Km} / \mathrm{sec}$. Many DM candidates have been proposed in the literature [5]. Studies of the formation of structures in the Universe, and the standard cosmology models indicate that most DM should be cold, i.e., it should be not relativistic at the onset of galaxy formations. This excludes light particles as neutrinos as possible candidates to contribute in a significant way to the DM.

Supersymmetry predicts many new supersymmetric particles, among whom those named neutralinos are the lightest super-particles predicted by the theory. The neutralino is an example of a "weakly interacting massive particle" (WIMP). Such particles have been for a long time the most promising DM candidates, and have been the targets of most experiments designed to carry out a direct search for DM. The current cross section sensitivity in DM experiments is as small as $10^{-8}$ pbarn in a mass range from a few $\mathrm{GeV}$ to a few 
$\mathrm{TeV}$ [4]. This sensitivity is already good enough to exclude a fraction of the regions predicted by supersymmetric models. LHC experiments could detect directly DM particles produced in $p$ - $p$ collisions. So far, however, there is no evidence of supersymmetric nor generic new particles in LHC data in the TeV range up to $\sqrt{s}=8 \mathrm{TeV}$.

According to a pessimistic point of view, those negative results could suggest that DM particles have only gravitational interactions. In this case, due to their very small cross section, a detection with particle detectors would be impossible. Another possibility is that DM particles could be much heavier than the few $\mathrm{TeV}$ mass reached by current experiments. The possible existence of super-heavy dark matter particles, sometimes called Wimpzillas, would have interesting phenomenological consequences, including a possible solution of the problem of cosmic rays observed above the GZK cutoff. The literature is full of exotic names for such particles: Q-balls, mirror particles, CHArged Massive Particles, (CHAMPs), self interacting dark matter, cryptons, super-weakly interacting dark matter, brane world dark matter, heavy fourth generation neutrinos, etc. (see the references listed in [5]). Even if strongly interacting, these objects could remain 'dark' due to their large mass-to-surface area ratio and correspondingly low number density required to explain the observed DM mass density.

More recently, composite objects consisting of light quarks in a color superconducting phase have been suggested. In addition, super-heavy DM antiquark nuggets could exist and could perhaps solve the matter-antimatter asymmetry [17]; the detection of such anti-quark nuggets by cosmic ray experiments is discussed in [20]. The energy loss predicted for super-heavy DM particles varies in different models, but it is likely that such particles could be confused with meteors, since the velocity, $270 \mathrm{~km} / \mathrm{sec}$, is in the high-end tail of the meteor velocity distribution.

Here, we will focus our attention only on one possible kind of very massive particle called "nuclearite". It consists of neutral matter including a strange quark among its constituents. We make this choice because nuclearites are an example of particles already searched for by other experiments, and for which we can be able to compute some expected performance improvements which should be possible using JEM-EUSO as a possible detector.

Nuggets of Strange Quark Matter (SQM), composed of approximately the same numbers of up, down and strange quarks could be the true ground state of quantum chromodynamics $[28,1]$. SQM nuggets could be stable for all baryon numbers in the range between ordinary heavy nuclei and neutron stars. They may have been produced in the early Universe. As the strange quark is massive compared to almost massless up and down quarks, surface tensions lead to the suppression of a few s quarks. Thus, SQM should have a relatively small positive electric charge compared to that of heavy nuclei [15]. Macroscopic quark nuggets, neutralized by captured electrons, are called nuclearites. Otherwise, and generally in the case of small baryon numbers $\left(A \leq 10^{6}\right)$, assumed to be quasi totally ionized, they will be called strangelets. There are several concerns about the SQM hypothesis; one was raised in 1999, when heavy-ion collisions 
between gold nuclei were produced at the Brookhaven National Laboratory (USA) and, more recently, before the LHC run with heavy ions: negative strangelets would attract a positive nucleus and could eat it, in a sequence that could end with the digestion of the whole planet. Fortunately, however, there are theoretical considerations suggesting that negative strangelets are unlikely to exist $[14,6]$.

According to [15] nuclearites are considered to be large strange quark nuggets, with overall neutrality ensured by an electron cloud which surrounds the nuclearite core, forming a sort of atom. Nuclearites with galactic velocities are protected by their surrounding electrons against direct interactions with the atoms they might hit.

As a consequence, the principal energy-loss mechanism for a nuclearite passing through matter is atomic collision. For a massive nuclearite the energyloss rate is:

$$
\frac{d E}{d x}=-A \rho v^{2}
$$

where $\rho$ is the density of the traversed medium, $v$ the nuclearite velocity and $A$ is its effective cross-sectional area. The effective area can be obtained by the nuclearite density $\rho_{N}$. For a small nuclearite of mass less than $1.5 \mathrm{ng}$, the cross-section area $A$ is controlled by its electronic atmosphere which is never smaller than $10^{-8} \mathrm{~cm}$ :

$$
A=\left\{\begin{array}{l}
\pi \cdot 10^{-16} \mathrm{~cm}^{2} \text { for } M<1.5 \mathrm{ng} \\
\pi\left(\frac{3 M}{4 \pi \rho_{N}}\right)^{2 / 3} \text { for } M>1.5 n g
\end{array}\right.
$$

where $\rho_{N}=3.5 \cdot 10^{14} \mathrm{~g} / \mathrm{cm}^{3}$ is the nuclearite density and $M$ its mass.

According to Eq. 1, nuclearites having galactic velocity and mass heavier than $10^{-14} \mathrm{~g}$ penetrate the atmosphere, while those heavier than $0.1 \mathrm{~g}$ pass freely though an Earth diameter. Eq. 1 has been used by [15] to compute the amount of visible light emitted in the atmosphere, assuming that the light is emitted as a black-body radiation from an expanding cylindrical thermal shock wave and to compute therefore the apparent magnitude as defined for meteors. It turns out that there are two important differences that can help to discriminate between nuclearites and meteors. The first one is that the amount of light emitted by nuclearites is proportional to the atmospheric density and therefore most of the light is emitted near the ground level; the second difference is that a nuclearite of mass bigger than $0.1 \mathrm{~g}$ can move upward and this is extremely unlikely for a meteor.

Nuclearites and similar particles, as for example neutral Q-ball[22], have been searched for using different approaches. The experiments can be characterized by the detection area (S) and by the minimum nuclearite mass that can be detected (Mth), usually computed for a speed of $270 \mathrm{~km} / \mathrm{sec}$. Many techniques, summarized in Table 1 have been used to detect nuclearites: acoustic emission due to the thermal shock in aluminum gravitational wave cylindrical 
Table 1 Experimental techniques, locations, representative experiments, sensitive area and nuclearite mass thresholds computed for $v=270 \mathrm{~km} / \mathrm{sec}$.

\begin{tabular}{ccccc}
\hline Technique & location & Experiment & $\mathrm{S}\left(\mathrm{m}^{2}\right)$ & Mth $(\mathrm{gr})$ \\
\hline \hline thermo-acoustic & sea level & {$[3]$} & $\sim 1$ & $10^{-9}$ \\
damage & mountain 5230 m a.s.l. & {$[11]$} & 427 & $5 \cdot 10^{-14}$ \\
light in oil & underground 3700 hg cm $\mathrm{cm}^{-2}$ & {$[2]$} & $\sim 700$ & $2 \cdot 10^{-10}$ \\
light in water & underwater 2500 hg cm & {$[24]$} & $\sim 10^{5}$ & $2 \cdot 10^{-10}$ \\
earth or moon-quakes & earth/moon inner & {$[19]$} & $\sim 10^{11}$ & $\sim 10^{4}$ \\
\hline
\end{tabular}

detectors, damages in plastic materials like CR39, Makrofol or Lexan, light emission in oil or sea water, seismic waves induced by big nuclearites. Due to the uncertainties in the energy losses it is important to have different techniques to detect such exotic particles. Table 1 lists the different techniques and a representative experiment of each technique. It is not aimed at being a full list of the experiments done so far to search nuclearites, but it is a reasonable summary of the state of the art in this field.

\section{General description of JEM-EUSO payload and focal plane assembly}

A general description of the JEM-EUSO telescope has already been given elsewhere in this volume. We recall here only the essential points related to the meteor and nuclearite discussion. The role of the JEM-EUSO telescope [21] is to act as an extremely-fast $(\sim \mu \mathrm{s})$ and highly-pixelized $\left(\sim 3 \times 10^{5}\right.$ pixels $)$ digital camera with a large aperture (a diameter of about $2.5 \mathrm{~m}$ ) and a wide field of view (FoV) of $60^{\circ}$. It works in near-UV wavelengths $(290-430 \mathrm{~nm})$.

The optics focuses the incident UV photons onto the focal surface. The focal surface detector converts incident photons into electric pulses. The electronics counts the number of pulses in time intervals of $2.5 \mu$ s (Gate Time Unit GTU) and records it. When a signal pattern is found, a trigger is issued. This starts a sequence which eventually transmits to the ground operation center the signal data recorded within (and surrounding) a selected pixel region.

The combination of 3 Fresnel lenses has an angular resolution of $0.07^{\circ}$. This resolution corresponds approximately to a linear size of $550 \mathrm{~m}$ on the ground beneath the ISS located at an altitude above ground of about $400 \mathrm{~km}$.

The Focal Surface (FS) of JEM-EUSO has a spherical shape of about $2.3 \mathrm{~m}$ in diameter with about $2.5 \mathrm{~m}$ curvature radius, and it is covered by $\sim 5,000$ multi-anode photomultiplier tubes (MAPMTs). The FS detector consists of Photo-Detector Modules (PDMs), each of which consists of 9 Elementary Cells (ECs). Each EC contains 4 units of MAPMT (Hamamatsu R11265-03-M64, 2 inches in size, with $8 \times 8$ pixels). A total of $137 \mathrm{PDMs}$ are arranged on the FS. A Cockcroft-Walton-type high-voltage supply is used to suppress power consumption, including a circuit to protect the photomultipliers from instantaneous bursts of light, like in the case of lightning or bright fireball phenomena. 
The FS electronics system records the signals of UV photons generated by EECRs successively in time. A new type of front-end ASIC has been developed for this mission, which has both functions of single photon counting and charge integration in a chip with 64 channels. The FS electronics is configured in three levels corresponding to the hierarchy of the FS detector system: frontend electronics at EC level, PDM electronics common to 9 EC units, and FS electronics to control 137 units of PDM electronics. Anode signals of the MAPMT are digitized and recorded in ring memories for each GTU to wait for a trigger assertion, then, the data are read and sent to control boards. JEMEUSO uses a hierarchical trigger method to reduce the huge original data rate of $\sim 10 \mathrm{~GB} / \mathrm{s}$ down to $297 \mathrm{kbps}$, needed to transmit data from the ISS to the ground operation center.

\section{Simulations}

\subsection{Meteor simulations}

A very simple model of meteor phenomena has been preliminarily developed in order to make it possible to carry out a campaign of numerical simulations aimed at analyzing the kind of signals which may be produced on the JEMEUSO focal plane in a variety of possible observing scenarios.

One thing to be taken into account, is that meteor phenomena are extremely variable in many important respects, including duration, brightness, brightness variation, speed and trajectory of the impacting body. Any model aimed at exploring the space of parameters, must therefore be quite flexible.

In a first preliminary version, the model assumes that a meteor phenomenon occurs at some instant, corresponding to a given initial height $h_{\text {beg }}$ of the meteoroid. A Cartesian reference system is then defined, having its $x, y$ plane on the ground, the origin on the point corresponding to the vertical projection of the meteoroid, which at the instant $t=0$ has therefore coordinates (in $\mathrm{km}$ ) $\left(0,0, h_{\text {beg }}\right)$. At the same time, the coordinates of the JEM-EUSO focal plane is assumed to be $\left(x_{0}, y_{0}, 400\right)$. For sake of simplicity, it is assumed that JEMEUSO has a constant height of $400 \mathrm{~km}$ (no vertical component of the motion). The direction of the $x$ axis is chosen to be coincident with the direction of the horizontal motion of JEM-EUSO, supposed to be linear and uniform. These assumptions are simplistic, but they are reasonable for describing events which have durations which do not exceed some seconds. The initial $x_{0}$ and $y_{0}$ coordinates are chosen randomly or selected by the operator, but in any case a control is done in order to exclude the possibility that the simulated event could be outside the cone of observability of JEM-EUSO.

The model assumes an initial velocity vector for the meteoroid, and a given total duration for the event. Both the velocity and the duration can be chosen randomly (within some ranges of realistic options) or decided by the operator.

The variation of the brightness of the meteor as a function of time, or meteor lightcurve, is also chosen randomly, or fixed according to the needs 

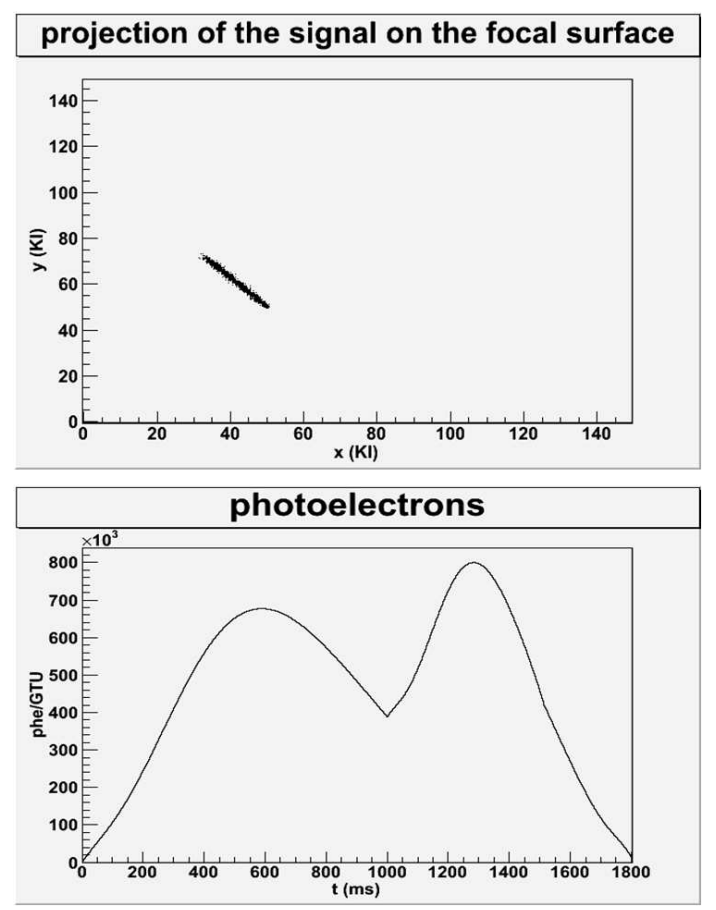

Fig. 3 Example of meteor signal computations carried out using the simulator described in the text. The top panel shows the resulting path of the signal in the focal plane, while the bottom panel shows the simulated lightcurve, including a secondary burst occurring at $t \simeq 1000 \mathrm{msec}$.

of the operator. Since the shape of the lightcurve can be very variable in the real world, the model adopts a very flexible approach, based on a polynomial of $9^{t h}$ degree. In most practical applications performed so far, some visual control has been done in order to select preferentially simulated lightcurves which look reasonably realistic, taking into account the large intrinsic variety of the phenomenon, as shown, for instance, in the analysis by [25]. Moreover, since it is known that real meteors can exhibit one or more secondary bursts, the model includes the possibility to simulate one secondary burst, occurring at some instant before the end of the event, and having a morphology which is again represented by a degree 9 polynomial (again, fixed or randomly chosen).

Having selected the above parameters, the computations executed by the numerical simulator are easy. At each time step, generally chosen to be equal to $10^{-3} \mathrm{sec}$, the position and brightness of the meteor are computed, and the fluxes reaching the JEM-EUSO focal plane, taking into account the varying distance from the detector, are then computed, the magnitudes being directly converted to photo-electrons per GTU. The varying position of the signal on the focal plane at different time steps is also computed. The same overall 
computation is also done at each time step for an hypothetical ground-based observer located in the origin of the coordinate system defined above.

In this way, a large variety of possible cases can be modeled. An example is given in Figure 3. The results of such simulations can be used to develop an overall strategy of JEM-EUSO observation of slow events. In particular, we have so far preliminarily analyzed the possibility to derive a correct identification of the events by adopting different triggering techniques, and considering meteor events having a variety of possible brightness, observed in different background conditions. By the way, the meteor simulator is also useful to simulate the signals produced on the focal plane by sources fixed on the ground, so mimicking what happens when urban agglomerations enter the field of view.

The simulation work carried out so far has been still fairly limited, but the results have been positive, and suggest that JEM-EUSO could be able to detect meteors down to absolute visual magnitudes of the order of $5-6$, a limit which in absolute terms is not better than the performances of the best ground-based facilities, but which becomes very interesting for meteor science by considering the large field of view and high duty cycle of the JEMEUSO detector. Moreover, the detection of faint meteors, though interesting and useful as it may be, is probably not as important as a systematic and complete detection of brighter events, including fireballs, which may be visible even in observing conditions far to be optimal for cosmic rays detection. For bright events, the possibility to have also simultaneous detections from groundbased meteor observing networks can be extremely useful to derive for these objects accurate $3 D$ trajectories, and to compute for them the original orbit, and possibly the coordinates of meteorite fall for objects sufficiently big and resistant to reach the ground.

In its current version, the meteor simulator developed for JEM-EUSO can be still largely improved. For instance, no meteor deceleration has been taken into account so far. Also the choice of a set of reference lightcurves to be preferentially adopted would deserve some further work. Moreover, it is known that the number of secondary bursts can well be higher than one in real events, and this also should be taken into account in future improvements of the model.

\subsection{Meteor detection simulations: triggering and recording}

The simulations of meteor events described above provide the input for the next necessary step of the analysis, namely a simulation of the response of the on-board electronics for the purposes of identification and recording.

Currently, the response of the detector, including optics and focusing, and the response of the photomultipliers in the focal surface, is parameterized. An overall throughput efficiency of $10 \%$ is assumed. An optical point spread function (PSF) of $\sim 2.5 \mathrm{~mm}$ is assumed. Cross-talk, pixel-to-pixel non-uniformity response in gain of the order of $10 \%$, as well as poissonian fluctuations of the night glow background are introduced in the simulations. The FS is considered to be a uniform layer of MAPMTs. 
Table 2 For different absolute magnitudes of meteors in visible light, the corresponding flux in the $U$-band are shown (according to the Flux Density Converter of the Spitzer Science Center; details can be found at the web site http://ssc.spitzer.caltech.edu/warmmission/propkit/pet/magtojy/index.html). The corresponding number of photons per second, the number of photo-electrons per GTU, the typical mass of the meteor, and the number of events expected to be observed by JEM-EUSO (the latter is computed assuming a duty cycle of 0.2 ) are also shown.

\begin{tabular}{cccccc}
\hline $\begin{array}{c}\text { magnitude } \\
(\mathrm{M})\end{array}$ & $\begin{array}{c}\text { U-band flux } \\
\left(\mathrm{erg} / \mathrm{s} / \mathrm{cm}^{2} / \mathrm{A}\right)\end{array}$ & $\begin{array}{c}\text { photons } \\
\left(\mathrm{s}^{-1}\right)\end{array}$ & $\begin{array}{c}\text { photo-electrons } \\
(\mathrm{GTU}=2.5 \mu \mathrm{s})^{-1}\end{array}$ & $\begin{array}{c}\text { mass } \\
(\mathrm{g})\end{array}$ & $\begin{array}{c}\text { collisions in } \\
\text { JEM-EUSO FoV }\end{array}$ \\
\hline 7 & $6.7 \cdot 10^{-12}$ & $4.3 \cdot 10^{7}$ & 4 & $2 \cdot 10^{-3}$ & $1 / \mathrm{s}$ \\
5 & $4.2 \cdot 10^{-11}$ & $2.7 \cdot 10^{8}$ & 23 & $10^{-2}$ & $6 / \mathrm{min}$ \\
0 & $4.2 \cdot 10^{-9}$ & $2.7 \cdot 10^{10}$ & 2300 & 1 & $0.27 /$ orbit \\
-5 & $4.2 \cdot 10^{-7}$ & $2.7 \cdot 10^{12}$ & $2.3 \cdot 10^{5}$ & 100 & $6.3 /$ year \\
\hline
\end{tabular}

Due to the fact that the meteor luminosity can span over several orders of magnitudes, different modes of detection must be designed, accordingly. In particular, both acquisitions in single photon counting mode and acquisitions in charge integration mode have to be planned. The single photon-counting technique has been developed mainly for cosmic rays observation, thus for cases in which the maximum intensity of the signal is expected to be of the order of few tenths of photo-electrons per pixel in one GTU. At higher photoelectron densities, the photon-counting technique becomes less accurate due to the pile-up, and charge integration is therefore needed. Table 2 summarizes the relation between meteor absolute magnitude, photon flux, number of photoelectrons at the maximum of the development, mass and expected number of events in the field of view of JEM-EUSO in nadir mode. One should note that the definition of absolute magnitude for meteors, namely the apparent brightness measured if the meteor was seen from the ground at a distance of $100 \mathrm{~km}$ along the vertical (toward the zenith) implies that the corresponding brightness as seen from JEM-EUSO turns out to be fainter, due to the fact that the ISS is located at an height of $400 \mathrm{~km}$. A meteor located at an height of $100 \mathrm{~km}$ above the ground will be seen from a distance of $300 \mathrm{~km}$ from JEMEUSO (assuming that the meteor has the ISS at the zenith). If the meteor has an absolute magnitude $M$, the corresponding apparent magnitude measured by JEM-EUSO will be therefore approximately $m_{r}=M+2.4$.

In case of particularly bright meteors, a switch logic implemented at high voltage level is needed in order to reduce the collection efficiency of the MAPMT, limiting the intensity of the signal. In this way, saturation problems are avoided and a wide dynamic range $\left(10^{6}\right)$ is guaranteed.

The most significant difference between single-photon counting and chargeintegration logics is the pixel size. In fact, in case of charge-integration, pixels are grouped in bunches of 8 units (named KIs) to reduce memory resources. Figure 4 shows the currently assumed KI grouping $(2 \times 4$ pixels $)$.

Despite the fact that at high (faint) magnitudes meteor signals are comparable in luminosity to air showers produced by cosmic rays, their time duration is by orders of magnitude longer: seconds compared to hundreds $\mu \mathrm{s}$ in 


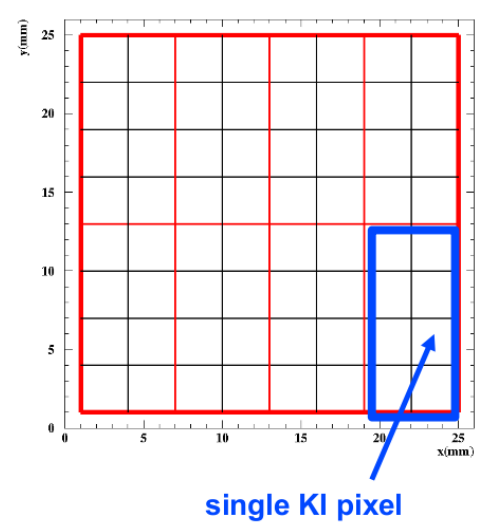

Fig. 4 A schematization of a M64 Hamamatsu MAPMT is presented. The red line represents the MAPMT contour. The black squares are the MAPMT pixels used in photoncounting mode. The blue contour shows the assumed dimension of the KI pixel, which is the pixel used in charge-integration mode.

case of air showers. Therefore, to collect and properly record meteor events, some trade-off is needed because of the extremely high sampling rate of JEMEUSO $(2.5 \mu \mathrm{s})$. Such high time resolution would be in itself quite interesting, because some events could be acquired at least partially with such a high time-resolution. However, as this is not strictly necessary, it is convenient to assume in a conservative way that the light profile has to be sampled in order to reduce the recorded signal size of the event at a level comparable to air shower events ( $\sim 300$ kbytes $)$.

In case of bright meteors, the acquisition is performed using only $288 \mathrm{KI}$ channels per PDM. This is a factor 8 smaller than photon-counting pixels (2304 channels) used for fainter meteors, so that a slightly denser sampling becomes possible. In case of KI we assume to acquire a total of 1024 GTUs sampled at a rate of one every 1024 GTUs $(=2.56 \mathrm{~ms})$, giving a corresponding total time of $1024 \times 1024$ GTU $=2.6 \mathrm{~s}$, which is in general sufficient to fully cover a typical meteor track. In this way, the data budget for one event is $\sim 300$ kbytes $(288$ channels $\times 1$ byte/channel $\times 1024$ GTUs). On the other hand, in case of high magnitude (fainter) events where photon-counting pixel mode is used, an equivalent solution is to acquire a total of $128 \mathrm{GTU}$ with a sampling rate of one every 4096 GTU $(=10.24 \mathrm{~ms})$ for a total of $128 \times 4096$ GTUs $=$ $1.3 \mathrm{~s}$. Even though the time span is shorter than in the case just seen above, it is in any case sufficient to collect the visible part of the track. Naturally, the performance would improve in case we could integrate the data in the GTUs instead of performing a sampling. This solution is under study, however, we consider here the most conservative case.

Of course, another important parameter is sky brightness. Moonlight is the largest background component. Figure 5 shows the observational duty cycle as a function of the accepted level of luminosity. In our study we assumed 


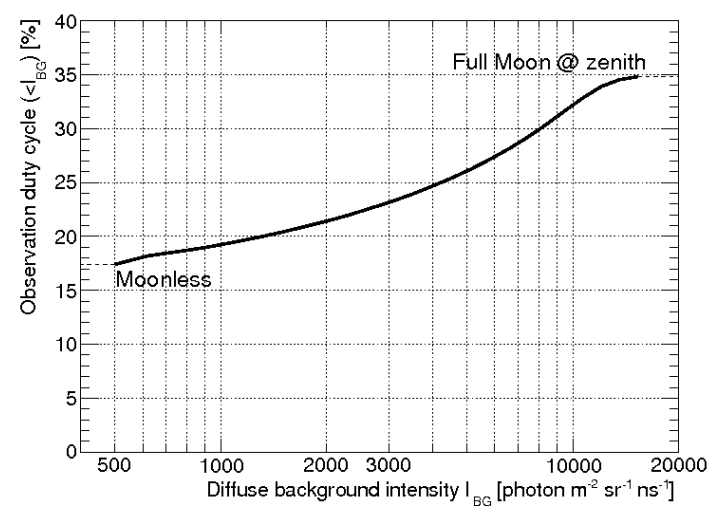

Fig. 5 Observational duty cycle $\eta$ as a function of threshold background level $I_{\mathrm{BG}}^{\mathrm{thr}}$.

three different possibilities. One case corresponds to conditions of almost new moon, namely a background of $\sim 750 \mathrm{ph} / \mathrm{m}^{2} / \mathrm{ns} / \mathrm{sr}$. The other two cases are characterized by higher background levels: $\sim 3000 \mathrm{ph} / \mathrm{m}^{2} / \mathrm{ns} / \mathrm{sr}$ and $\sim 7500$ $\mathrm{ph} / \mathrm{m}^{2} / \mathrm{ns} / \mathrm{sr}$.

The trigger logic is defined following an approach similar to that adopted for air showers (cosmic rays) events. A detailed explanation of the trigger strategy for air showers is described in [10]. In the case of meteors, the first trigger level is issued if for few consecutive GTUs, one KI channel detects an excess of signal, and such an excess, integrated along consecutive GTUs, is higher than a preset threshold. The threshold is set at a level such that Poissonian fluctuations of the background rarely mimic this pattern, that is at a level of $\sim 1 \mathrm{~Hz}$ on the entire FS.

In case of much brighter meteors, the signal becomes too intense and it is necessary to reduce the collecting efficiency of the PMTs to avoid saturation problems. By means of a switch logic, if the current produced at the anode is higher than a preset value, the collection efficiency on the first dynode is reduced in consecutive steps, each one corresponding to a factor of 100 . The information about the status of the switches is kept in memory in order to re-scale the data obtained in different conditions. In this way, the dynamic range of the measurement can be enlarged to cover 6 orders of magnitudes in luminosity, corresponding to a range of 15 magnitudes.

\subsection{The discrimination of anthropogenic lights}

According to previous studies, city lights look as objects of magnitude $M>2$. As explained in section 6 , this class of sources will be detected by JEM-EUSO. Therefore, some criteria have to be established in order to exclude them from automated triggering procedures. Due to the ISS orbital velocity of $\sim 7 \mathrm{~km} / \mathrm{s}$, a

fixed luminous spot on the Earth's ground produces a luminous track moving at the ISS speed, but in the opposite direction. As a first approximation, also 


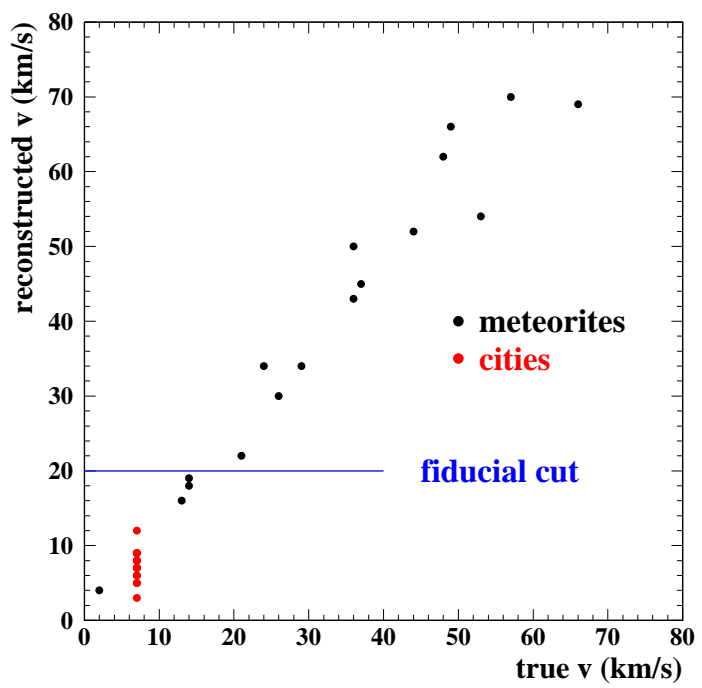

Fig. 6 Scattered plot showing the projections on the FS of the true and reconstructed velocities for simulated meteors (black dots) and cities (red dots). A fiducial line to avoid triggering on cities based on the reconstructed projection of the velocity is also shown.

airplanes will exhibit a similar behaviour, as their speed is less than $0.3 \mathrm{~km} / \mathrm{s}$. A second trigger level is therefore needed to provide some discrimination of anthropogenic sources. Being adapted from the procedure of detection of air showers produced by cosmic rays, which basically follows the luminous spot on the PDM, the trigger logic can make a first guess of the apparent velocity and direction of the motion on the focal surface, in order to verify whether it is compatible with a static source located on the ground.

In order to check this possibility, we simulated a sample of meteors having magnitude $M=2$ and different velocities on the FS, and a sample of cities having different radii $(0<\mathrm{R}<10 \mathrm{~km})$ and a variety of magnitudes $(2.5<\mathrm{M}$ $<4)$. Figure 6 shows a plot of the "true", velocities of simulated meteors and cities, and the velocities reconstructed by the trigger logic. It is easy to see that, on one hand, the procedure of measurement of the source velocities works reasonably well. Moreover, in general, cities, regardless of their brightness and size, exhibit low velocities. Therefore, a fiducial threshold can be placed on the reconstructed velocity at trigger level, to prevent from issuing a trigger driven by the detection of such low-velocity sources. In the specific case shown in the Figure, the fiducial line has been placed at $20 \mathrm{~km} / \mathrm{s}$, to be conservative. Of course, this threshold could be relaxed when flying above oceans, where the density of anthropogenic lights in the FoV is expected to be much smaller. Finally, this cut in velocity will be applied only for the range of meteorite magnitudes compatible with city lights, whose flux is anyway sufficiently high 
$(\sim 1 \mathrm{ev} / \mathrm{h}$ in JEM-EUSO FoV). For much brighter objects, the recorded signal will be much higher and the above fiducial threshold will not be required.

\section{Preliminary results}

A very simplified reconstruction algorithm has been applied to simulated meteor profiles in order to check the expected performances of the system, in particular for what concerns the expected magnitude threshold for detection of these events in different background conditions, by applying consistently either the analog or digital acquisition mode. A more refined technique is expected to be applied in the future, in such a way as to allow us to improve the present results, which can be considered, therefore, as fairly conservative. In the present approach, the signal from each pixel is acquired assuming to use the sampling technique explained in the previous Section, taking into account the detector response and background contribution. Three different photo-electron levels $(16,64,160$ photo-electrons/pix/GTU) corresponding to the background intensities of 750,1500 and 7500 photons $/ \mathrm{m}^{2} / \mathrm{sr} / \mathrm{ns}$ have been considered.

The procedure explained below assumes to use KI pixels, but the same approach has also been applied to the case of digital pixels. First of all, the search of the meteor track on the FS is performed by selecting within each GTU the KI pixel with the highest signal. Figure 7 shows as an example the selected pixels in the entire time span of a simulated meteor track having a magnitude $M=2$, as well as the original track. During the brightest part of the meteor track, the right pixels are selected and they look aligned on a track as expected. On the other hand, in the first part of the meteor lightcurve, where the signal is still much fainter and background fluctuations dominate, pixels scattered over the entire portion of FS under consideration are selected. As a second step, a fit is operated on the detected part of the track, and then extrapolated to the rest of the track embedded in the noise. The average background level is then subtracted to all pixels.

Figure 8 shows the original light profile of the meteor, and the signal extracted using the reconstruction technique that we have just described. In order to determine the highest magnitude level at which the meteor profile could be reconstructed with reasonable uncertainties we have defined the following criteria:

$$
\begin{gathered}
\Delta I=\frac{\int\left(\frac{d p h e}{d t}\right)_{\text {rec }} \cdot d t-\int\left(\frac{d p h e}{d t}\right)_{\text {true }} \cdot d t}{\int\left(\frac{d p h e}{d t}\right)_{\text {true }} \cdot d t} \cdot 100(\%), \\
\Delta M a x=\frac{\text { Max }_{\text {rec }}-\text { Max }_{\text {true }}}{\text { Max }_{\text {true }}} \cdot 100(\%),
\end{gathered}
$$

where $\Delta I$ provides the resolution on the integrated lightcurve, while $\Delta M a x$ gives an information on the uncertainty of the estimation of the peak of the light profile which is proportional to the magnitude of the event. The condition 

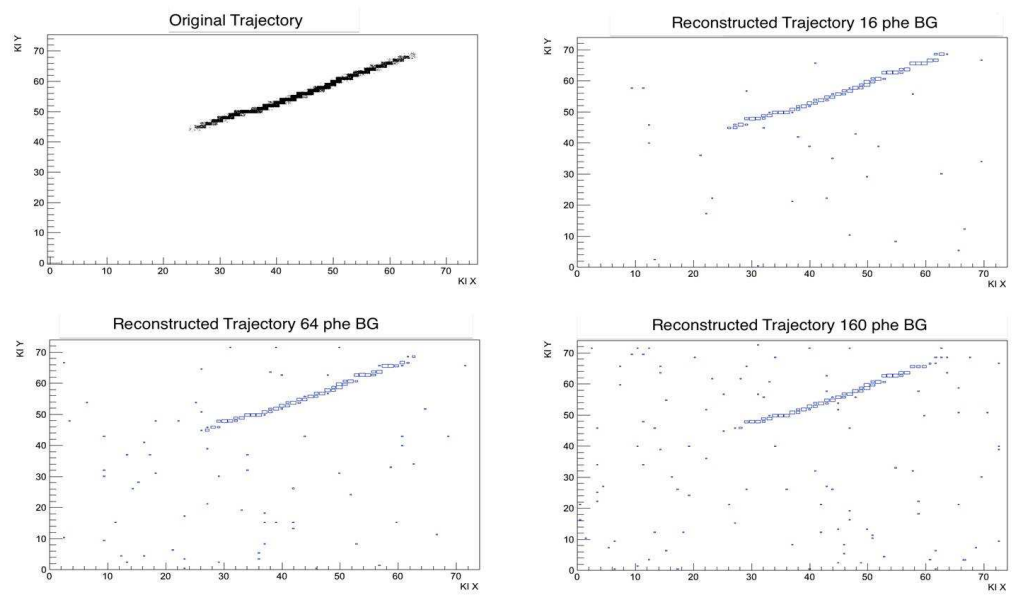

Fig. 7 Original trajectory of a meteorite of magnitude $\mathrm{M}=2$ at pupil level, and the portion of the trajectory selected by the simple reconstruction technique described in the text for different background levels. For low background levels a longer track is reconstructed, as expected.
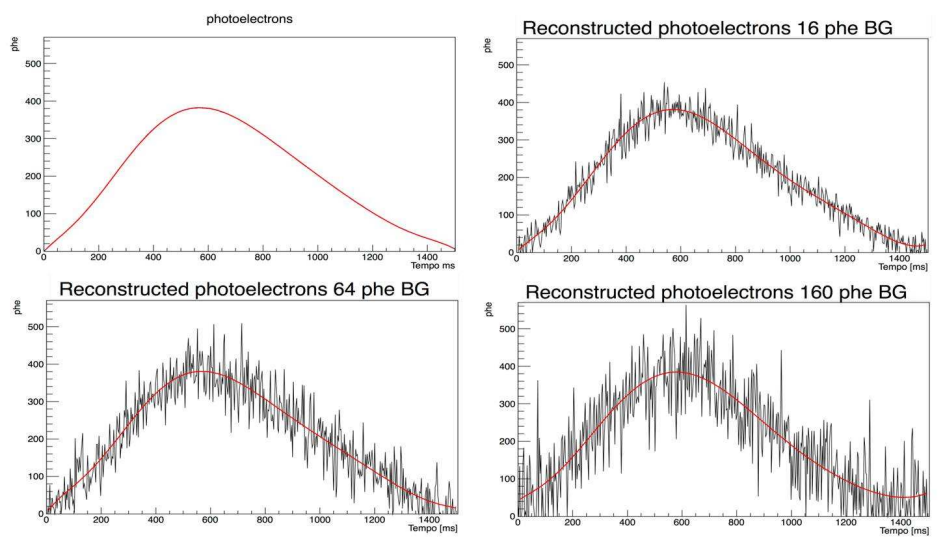

Fig. 8 Original light profile of a meteor of magnitude $M=2$ at pupil level, and the signal detected at FS level assuming different background levels as explained in the text.

is that both the above parameters are reconstructed with uncertainties $\Delta I$, $\Delta$ Max $<20 \%$. A quality cut on the shower profile is applied as well, to remove unsatisfactory reconstructions according to the above criteria.

Table 3 summarizes the results obtained for different background levels and magnitudes. This result shows that using KI information the threshold for meteor observation is around magnitude 4 for low background levels and increases up to magnitude 2 for very high background conditions. Interestingly, we see that it is possible to detect and properly reconstruct meteor profiles at $M=+4$, for which the photon-counting technique starts suffering from 
Table 3 Uncertainty on the reconstruction of $\Delta I$ and $\Delta M a x$ for meteors of different magnitude and various background levels.

\begin{tabular}{cccc}
\hline \hline $\begin{array}{c}\text { Background } \\
\text { (phe/KI-pixel/GTU) }\end{array}$ & magnitude & $\begin{array}{c}\Delta I \\
(\%)\end{array}$ & $\begin{array}{c}\Delta \text { Max } \\
(\%)\end{array}$ \\
\hline 16 & -1 & -0.2 & 0 \\
16 & 0 & -0.1 & -0.4 \\
16 & 1 & -1.6 & -1.1 \\
16 & 2 & -5.7 & -3.5 \\
16 & 3 & -0.1 & -2.2 \\
16 & 4 & -19.5 & -10.7 \\
\hline 64 & -1 & 0.7 & 0.3 \\
64 & 0 & -0.2 & -0.4 \\
64 & 1 & 3.9 & 1.7 \\
64 & 2 & 0.7 & -0.4 \\
64 & 3 & 11.3 & 5.0 \\
\hline 160 & -1 & -1.1 & -0.8 \\
160 & 0 & -7.5 & -3.9 \\
160 & 1 & 5.8 & 3.2 \\
160 & 2 & 1.9 & 0.5 \\
\hline \hline
\end{tabular}

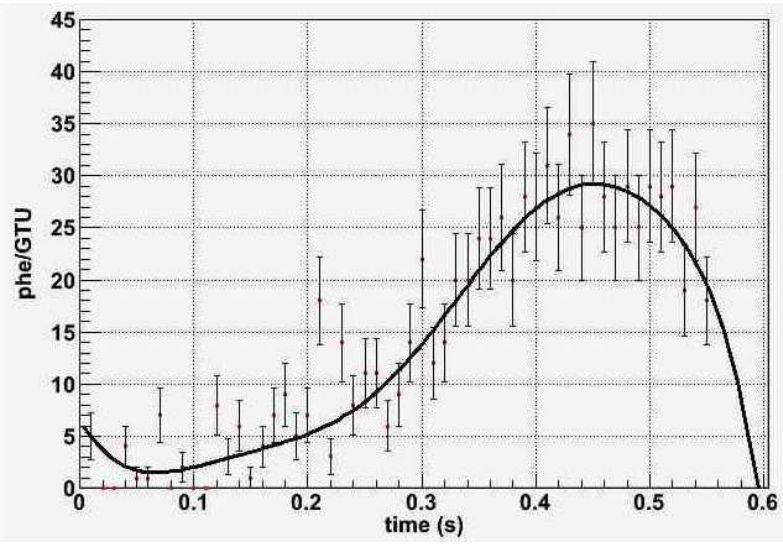

Fig. 9 Reconstructed light profile of a meteor of magnitude $M=+5$ at pupil level detected in photon-counting mode.

pileup (at the peak, $\sim 70$ phe/pixe/GTU are expected according to Table 2). Moreover, as the expected number of events in the FoV of JEM-EUSO is of the order of $\sim 1 /$ min at this magnitude, it would be possible to record many of them during dark nights. Longer exposure time is needed for brighter meteors such as $\mathrm{M}=+2$ where the expected flux is expected to be of $\sim 1 \mathrm{ev} / \mathrm{h}$.

For meteors of magnitude $\mathrm{M}>4$ photon-count mode is needed, rather than charge integration (KI), because the smaller size of pixels improves the signal over noise ratio, and photon-counting mode becomes more reliable. Figure 9 shows an example of reconstruction of the signal of a meteorite of $M=+5$ obtained by adopting the photon-counting sampling mode as described in the 
previous Section. The reconstructed meteor lightcurve turns out to be a quite reasonable fit of the "true", simulated signal. A more detailed study indicates that the threshold level is around $M=+5.5$ if the sampling technique described in Section 5.2 is adopted. We have also seen in our simulations that, by using a $25 \mu \mathrm{s}$ GTU, it would be possible to push the sensitivity threshold for meteors around $\mathrm{M}=+7$.

\subsection{Nuclearites}

Although a dedicated simulation of the signals produced by nuclearites moving through the atmosphere would be needed for a detailed assessment of the expected performances of JEM-EUSO in detecting and recording these events, the results already obtained for meteors can be used to draw some general and preliminary conclusions.

First of all, the detection sensitivity to nuclearites can be extrapolated from previous results obtained for the meteors. In particular, the visual magnitude of an atmospheric nuclearite located at a distance $h$ from an observer can be computed according to [15] as:

$$
M=10.8-1.67 \cdot \log _{10}(m / 1 \mu g)+5 \cdot \log _{10}(h / 10 k m) .
$$

By inverting equation 5 it is possible to estimate the minimum mass of the nuclearite detectable as a function of the distance of the object and its mass. In the following, the magnitude is meant to be the absolute one. Results indicate that JEM-EUSO is sensitive to objects having mass $m>10 \mathrm{~g}$ when working in single photon-counting mode, and to $m>100-1000 \mathrm{gr}$ when working in charge integration mode. There is of course a dependence upon the sky background luminosity (mainly due to Moon phase). According to [15] the upper limit to the altitude $\left(h_{\max }\right)$ at which nuclearites effectively generate light is described by the following relation:

$$
h_{\max }=2.7 \cdot \ln \left(\mathrm{m} / 1.2 \cdot 10^{-5} \mathrm{~g}\right) \mathrm{km} .
$$

Thus, for the range of masses mentioned above, $h_{\max }$ is expected to be located between $36 \mathrm{~km}$ and $60 \mathrm{~km}$.

For what concerns the triggering strategy to be adopted for these events, the same algorithms already developed for meteors can be used, simply varying the total sampling time in order to take into account the shorter duration of the phenomenon (and correspondingly shorter track length). Assuming to be in most unfavorable conditions, namely a nuclearite starting to emit at an height of $60 \mathrm{~km}$, and moving along a trajectory having a zenith angle such that the track crosses the entire PDM along its diagonal $(\sim 42 \mathrm{~km})$ before landing at ground, and taking into account a velocity of $250 \mathrm{~km} / \mathrm{s}$, it follows that the total duration of the phenomenon is only $\sim 0.3 \mathrm{~s}$. Therefore, in charge integration mode (KI mode) the optimized condition would be to record the signal during 1024 GTUs, sampled at a rate of one every 128 GTUs, whereas 


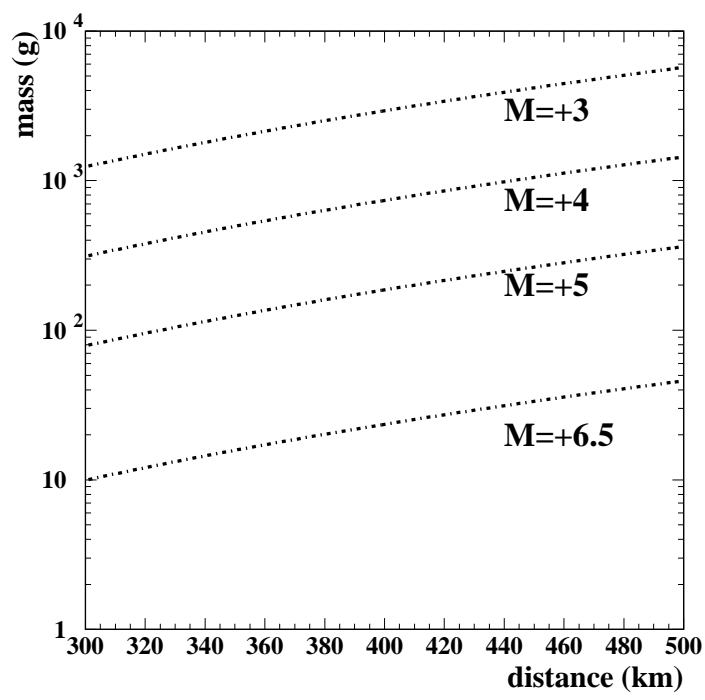

Fig. 10 Minimum size (expressed in mass) of the nuclearite detectable by JEM-EUSO as a function of its distance and for different thresholds in magnitude.

in single photon counting mode, one should record 128 GTU, with a sampling rate of one every 1024 GTUs. In both cases the total integrated time is $\sim 0.33$ $\mathrm{s}$ (one GTU being equal to $2.5 \mu \mathrm{s}$ ).

The easiest way to distinguish nuclearites from meteors is based on their velocity. Meteors have much slower speeds (in general below $72 \mathrm{~km} / \mathrm{s}$ ). As it is shown in Figure 6, already at trigger level it is possible to estimate the projected velocity of the signal on the FS with reasonable uncertainty. A subsequent data analysis of the recorded signals carried out later on the ground will certainly provide much more accurate results. Although it is not possible to derive directly from the data the $3 \mathrm{D}$ velocity vector of the source, a limit can be set to the recorded projected velocity $\left(v_{p r o j}\right)$. By requiring that $v_{p r o j}>v_{p r o j}^{\min }$ and assuming that the velocity of the nuclearite is $v=250 \mathrm{~km} / \mathrm{s}$, choosing a value for $v_{p r o j}^{\text {min }}$ automatically sets a limit on the zenith angle of the track $\theta_{\min }=\arcsin \left(v_{\text {proj }}^{\min } / v\right)$ and relative acceptance $R_{a c c}=\left(1+\cos \left(2 \cdot \theta_{\min }\right)\right) / 2$. Table 4 shows the relative acceptance as a function of different possible choices of $v_{\text {proj }}^{\min }$. It turns out that even a very tight cut on $v_{p r o j}^{\min }>160 \mathrm{~km} / \mathrm{s}$ makes the acceptance to decrease only by about a factor of 2 .

Another important fact to be taken into account is that nuclearites tend to develop at lower heights in the atmosphere compared to meteors. Moreover, any possible evidence of tracks moving upwards would be a clear sign of a nuclearite.

We can expect therefore that JEM-EUSO will be able to set very stringent limits on the flux of nuclearites, even after short acquisition times, due to 
Table 4 Impact on the relative acceptance $\left(R_{a c c}\right)$ (see text) as a function of different possible choices of $v_{\text {proj }}^{\min }$.

\begin{tabular}{ccc}
\hline \hline $\begin{array}{c}v_{\text {proj }}^{\min } \\
(\mathrm{km} / \mathrm{s})\end{array}$ & $\begin{array}{c}\theta_{\min } \\
(\mathrm{deg} .)\end{array}$ & $\begin{array}{c}R_{a c c} \\
(\%)\end{array}$ \\
\hline 100 & 23.6 & 84 \\
130 & 31.3 & 73 \\
160 & 39.8 & 59 \\
190 & 49.5 & 42 \\
220 & 61.6 & 23 \\
\hline
\end{tabular}

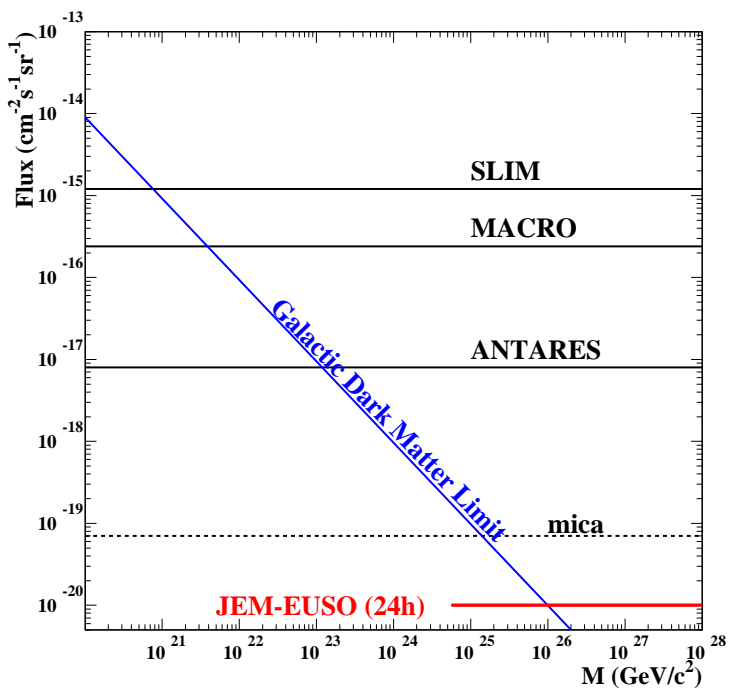

Fig. 11 The JEM-EUSO 90\% confidence level upper limit on the flux of nuclearites resulting from null detection over 24 hours of JEM-EUSO operations. The limits of other experiments [2], [11], [24], [26] are also shown for a comparison. The old mica limits [26] are dependent from several additional assumptions, respect to the other experiments.

the the tremendous instantaneous exposure $\left(\mathrm{A} \sim 5 \times 10^{20} \mathrm{~cm}^{2} \mathrm{~s} \mathrm{sr}\right)$ of the instrument. Even adopting a very severe rejection criterion, such as $v_{p r o j}^{\min }>$ $190 \mathrm{~km} / \mathrm{s}$, from Table 4 we can infer that for a $24 \mathrm{~h}$ accumulation time, a null detection would set a limit in flux at the $90 \%$ confidence level of the order of $10^{-20} \mathrm{~cm}^{-2} \mathrm{~s}^{-1} \mathrm{sr}^{-1}$ (see Figure 11).

\section{Conclusions and Perspectives}

Our preliminary analysis shows that JEM-EUSO has the capability to observe meteors down to magnitude $\mathrm{M}<5-6$, which could be lowered down to $\mathrm{M}<$ 7 using a $25 \mu$ s GTU. Taking advantage of its large field of view and high 
detection rate, JEM-EUSO is able to record a statistically significant flux of meteors as a function of their magnitudes, both for what concerns sporadic meteors, and in cases of different meteor swarms. Not suffering the constraints due to weather conditions, which limit the effectiveness of ground-based meteor observation networks, JEM-EUSO can become a very important facility in the field of meteor studies. A particularly important role can be played in the detection of bright meteors and fireballs, because these events can be detectable even in very unfavorable conditions of sky background, therefore a monitoring of these events can be always active, whereas the detection of faint meteors requires more optimal observing conditions, when the primary activity of the detectors will be devoted to the observation of extremely energetic cosmic rays.

An exciting development to be explored is the possibility that bright meteors might have in some cases some signal persistence. In the simulations carried out so far, it has been assumed that the signal is instantaneous and lasts for a time much shorter than the time step adopted in the simulations $(0.001 \mathrm{sec})$. However, it is possible that in the case of bright meteors this could be not completely realistic. In the case that a meteor is so bright to produce along its trajectory some signals lasting for some non-negligible time, it might be possible in principle to exploit the movement of the ISS in order to derive at least some rough $3 \mathrm{D}$ reconstruction of the event, leading to a computation of the position and velocity vectors, instead of a $2 \mathrm{D}$ simple projection of the motion. In the case that this could be shown to be possible, this would be a strong improvement, though forcedly limited to a small fraction of bright events.

Our preliminary analysis concerning the possible detection of nuclearites indicate that JEM-EUSO will be sensitive to nuclearites with mass higher than a few $10^{24} \mathrm{GeV} / \mathrm{c}^{2}$ and will be able, after a run time of only $24 \mathrm{~h}$, to provide limits on nuclearite flux lower by one order of magnitude with respect to the limits of the experiments carried out so far.

\section{Acknowledgements}

This work was partially supported by the Italian Ministry of Foreign Affairs, General Direction for the Cultural Promotion and Cooperation. Discussions with some meteor experts, including Jiri Borowicka, Pete Jenniskens and Jeremy Vaubaillon were helpful and stimulating.

\section{References}

1. Alcock, C. and Olinto A., Exotic Phases of Hadronic Matter and their Astrophysical Application, Ann. Rev. Nucl. Part. Sci. 38 161-184 (1988).

2. Ambrosio, M., et al. (MACRO Coll.), Nuclearite Search with the MACRO detector at Gran Sasso, Eur. Phys. J. C, 13, 453-458 (2000)

3. Astone P. et al., et al., Upper limit for nuclearite flux from the Rome gravitational wave resonant detectors, Phys. Rev. D 47 4770-4773 (1993). 
4. Baudis L., talk at the Neutrino 2012 conference Kyoto Japan (2012)

5. Bertone G,, Hooper D. and Silk J., Particle dark matter: Evidence, candidates and constraints, Phys. Rept. 405 279-390 (2005) [hep-ph/0404175].

6. J. P. Blaizot, J. Iliopoulos, J. Madsen, G. G. Ross, P. Sonderegger, H. J. Specht, CERN2003-0001 (2003).

7. Bottke, W. F., et al., Asteroids III, Univ. of Arizona Press, Tucson, p.395 (2002).

8. Burbine, T. H., et al., Asteroids III, Univ. of Arizona Press, Tucson, p. 653 (2002).

9. Adams Jr., J.H., et al. (JEM-EUSO Coll.), An evaluation of the exposure in nadir observation of the JEM-EUSO mission, Astroparticle Physics, 44, 76-90 (2013)

10. Catalano, O., et al. (JEM-EUSO Coll.), The trigger system of the JEM-EUSO Telescope, Proc. $31^{\text {th }}$ Int. Cosmic Ray Conference, 5, 1049-1052 (2009).

11. Cecchini, S., et al. (SLIM Coll.), Results on the search for strange quark matter and Q-balls with the SLIM experiment, Eur. Phys. J. C, 57, 525-533 (2008).

12. Ceplecha, Z., et al., Meteor Phenomena and Bodies, Space Science Reviews, 84, 327-471 (1998).

13. Ceplecha, Z., et al., Meteoroids 1998, Astron. Inst. Slovak Acad. Sci., Bratislava, 37 (1998).

14. Dar A.,De Rujula A. and Heinz U W, Will relativistic heavy ion colliders destroy our planet?, Phys. Lett. B 470 142-148 (1999).

15. De Rujula, A. and Glashow, S.L., Nuclearites - a novel form of cosmic radiation, Nature, 312, 734-737 (1984).

16. Ebisuzaki, T., et al. (JEM-EUSO Coll.), The JEM-EUSO Project: Observing Extremely High Energy Cosmic Rays and Neutrinos from the International Space Station, Nuclear Physics B (Proc. Suppl.), 175, 237-240 (2008).

17. Gorham, P., W., Antiquark nuggets as dark matter: New constraints and detection prospects, Phys. Rev. D 86, 123005/1-8 (2012)

18. Gruen, H., et al., in: Properties and interactions of interplanetary dust; Proceedings of the Eighty-fifth Colloquium, Marseille, France, July 9-12, 1984 (A86-42326 20-90). Dordrecht, D. Reidel Publishing Co., 105 (1985).

19. Herrin E. T., Rosenbaum D. C. Teplitz V. L., Seismic search for strange quark nuggets, Phys. Rev. D 73 043511/1-7 (2006). [astro-ph/0505584].

20. Lawson K., Quark Matter Induced Extensive Air Showers, Phys. Rev. D 83 103520/1-9 (2011).

21. Kajino, F. et al., The JEM-EUSO mission to explore the extreme Universe, Nuclear Instruments and Methods A, 623, 422-424 (2010).

22. Kusenko A., Kuzmin V., Shaposhnikov M.E. and Tinyakov P. G., Experimental signatures of supersymmetric dark matter Q balls, Phys. Rev. Lett. 80 3185-3188 (1998). [hep-ph/9712212].

23. Morbidelli, A., Asteroids III, Univ. of Arizona Press, Tucson, p. 409 (2002).

24. Pavalas, G. E., et al. (ANTARES Coll.), Search for massive exotic particles with the ANTARES neutrino telescope, Proceedings of the $23^{\text {rd }}$ European Cosmic Ray Symposium, Moscow, 543 (2012).

25. Pecina, P. G. and Koten P. On the theory of light curves of video-meteors, Astronomy \& Astrophysics, 499, 313-320 (2009).

26. Price, P. B., Limits On Contribution Of Cosmic Nuclearites To Galactic Dark Matter, Phys. Rev. D, 38, 3813-3814 (1988).

27. Takahashi, Y., et al., The JEM-EUSO mission New Journal of Physics, 11, 065009/1-21 (2009)

28. Witten E., Cosmic Separation of Phases, Phys. Rev. D 30 272-285 (1984).

29. Zigo, P., et al., The activity and mass distribution of the Geminid meteor shower of 1996-2007 from forward scatter radio observations, Contrib. Astron. Obs. Skalnaté Pleso 39, 5-17 (2009). 